\section{Uso de métodos contraceptivos entre mulheres com vida sexual ativa em São Leopoldo, Rio Grande do Sul, Brasil}

\author{
Use of contraceptive methods by sexually active \\ women in São Leopoldo, Rio Grande do Sul, Brazil
}

\author{
1 Centro Universitário \\ do Vale do Taquari, \\ Lajeado, Brasil. \\ 2 Faculdade de Medicina, \\ Universidade Federal \\ de Pelotas, Pelotas, Brasil. \\ 3 Programa de Pós- \\ graduação em Saúde \\ Coletiva, Universidade \\ do Vale do Rio dos Sinos, \\ São Leopoldo, Brasil. \\ Correspondência \\ J. S. Dias-da-Costa \\ Departamento de Medicina \\ Social, Faculdade de \\ Medicina, Universidade \\ Federal de Pelotas. \\ Av. Duque de Caxias 250, \\ Pelotas, RS \\ 96030-002, Brasil. \\ jcosta@epidemio-ufpel.org.br
}

\begin{abstract}
In 2003, a population-based cross-sectional study was conducted in the urban area of São Leopoldo, Rio Grande do Sul, Brazil. The sample included 867 sexually active women from 20 to 60 years of age. The objective was to describe the use of contraceptive methods. Data were collected using a standardized questionnaire. The study analyzed the prevalence of contraceptive use and socioeconomic variables in women reporting an active sex life (84.5\%), stratified by age groups. Some 627 (61.1\%) women reported use of contraceptive methods. In the 20-49-year old group, $48.8 \%$ reported using oral contraceptives, $18.7 \%$ tubal ligation, $17.3 \%$ condoms, and 7.3\% IUDs. In the 50-60-year old group, the most widely used method was tubal ligation (79.6\%). Regarding tubal ligation, the schooling variable showed a linear trend, that is, women with less schooling showed a higher prevalence. Prevalence of oral contraception was higher in low-income women.
\end{abstract}

Contraceptives; Reproductive Control Agents; Oral Contraception; Tubal Sterilization
Ioná Carreno ${ }^{1}$

Juvenal Soares Dias-da-Costa 2,3

Maria Teresa Anselmo Olinto 3

Stela Meneghel 3

\section{Introdução}

No Brasil, em 1983, foi implantado o Programa de Assistência Integral à Saúde da Mulher (PAISM), com o objetivo de normatizar ações voltadas para cada etapa do ciclo vital feminino, integrando os princípios da atenção preventiva e dos cuidados curativos. O programa incluiu, entre outras, atividades de planejamento familiar. Esse enfoque diferenciado na saúde reprodutiva baseado na atenção integral à saúde superou as suspeitas acerca de intenções de controle da natalidade 1 .

O planejamento familiar é um conceito que agrega a decisão do casal quanto ao momento próprio para o início da procriação, do número de filhos e do intervalo entre eles 2 . Na Constituição Federal promulgada em 1988, o planejamento familiar foi considerado como livre decisão do casal, cabendo ao Estado e ao sistema de saúde garantirem o acesso à informação e aos métodos, sendo os direitos sexuais e reprodutivos garantidos para que o casal decida livremente com orientação e acompanhamento dos serviços de saúde.

Em 1994, na Conferência Internacional sobre População e Desenvolvimento, foi intensificado o interesse mundial nas políticas e programas reprodutivos. Tais ações devem ser baseadas na crença da necessidade de empoderamento individual, principalmente das mulheres, através de sua educação, na salvaguarda de 
sua saúde sexual e reprodutiva e capacitando os casais e os indivíduos a assumirem escolhas livres e informadas sobre a reprodução. Assim, firmou-se e ampliou-se o conceito de planejamento familiar, principalmente por considerar não só a mulher, mas também por incluir o papel do homem nas questões reprodutivas 3 .

Os métodos contraceptivos são os aliados do casal/parceiros para alcançar sucesso no planejamento familiar, evitando gravidezes não desejadas com seu uso adequado, além dos preservativos proporcionarem a prevenção de doenças sexualmente transmissíveis e da AIDS. Existem inúmeros métodos contraceptivos, mas sabe-se que, no Brasil, há hegemonia de dois métodos em especial, a anticoncepção oral e a ligadura tubária.

A Pesquisa Nacional sobre Demografia e Saúde, em 1996, relatava que 55\% das mulheres, no Brasil, utilizavam algum método contraceptivo. Nesse estudo, o método mais utilizado foi a ligadura tubária, aproximadamente $40 \%$ das mulheres referiam o uso desse método. Porém, na Região Sul do Brasil, os anticoncepcionais orais eram os métodos mais prevalentes, atingindo $34,1 \% 4$.

Do ponto de vista da saúde coletiva, o conhecimento do padrão de consumo dos métodos contraceptivos e das características das usuárias pode subsidiar as políticas públicas quanto à adequação da utilização e da disponibilidade dos meios para a população 5,6.

Assim, este estudo teve como objetivos descrever o padrão de consumo dos métodos contraceptivos e algumas características das usuárias em São Leopoldo, Rio Grande do Sul, Brasil, no ano de 2003.

\section{Materiais e métodos}

A Universidade do Vale do Rio dos Sinos (UNISINOS), por intermédio de um grupo de pesquisadores, elaborou um projeto de pesquisa intitulado Condições de Saúde das Mulheres: Estudo de Base Populacional na Região do Vale do Rio dos Sinos. Essa investigação foi um estudo transversal de base populacional que incluiu mulheres de 20 a 60 anos residentes na zona urbana da Cidade de São Leopoldo. O projeto foi submetido e aprovado pelo Comitê de Ética e Pesquisa da Faculdade de Medicina, Universidade Federal de Pelotas.

O objetivo do presente estudo foi analisar o consumo de métodos contraceptivos entre as mulheres de 20 a 60 anos de idade. A prevalência de uso foi realizada entre as mulheres que mencionaram vida sexual ativa.
A amostra inicial foi estimada levando-se em consideração alguns parâmetros incluídos no estudo, nível de confiança de $95 \%$, poder de $80 \%$, razão de não expostos: expostos, estabelecida em 1:3 (baseada na distribuição de classe social encontrada em Pelotas) 7 , razão de prevalência de 2,0. Adicionaram-se, ao tamanho da amostra, mais $10 \%$ de possíveis perdas e recusas e $15 \%$ para controlar fatores de confusão na análise dos dados.

Foi realizada uma amostra por conglomerados, sendo sorteados quarenta setores censitários entre os 270 existentes na zona urbana da Cidade de São Leopoldo. Cada um desses setores teve o primeiro quarteirão sorteado juntamente com o ponto de partida, garantindo-se aleatoriedade na coleta de dados. Dessa forma, para completar a população necessária, foram visitados 36 domicílios em cada setor.

Foram utilizados questionários padronizados, pré-codificados e pré-testados. O grupo de entrevistadoras foi composto por acadêmicas de cursos na área da saúde da UNISINOS, treinadas para a aplicação do instrumento. Também foi realizado teste piloto para treinamento das entrevistadoras, e os dados foram coletados entre março e dezembro de 2003.

O controle de qualidade foi realizado em uma amostra aleatória de $10 \%$ das pessoas incluídas no estudo, com o intuito de avaliar a qualidade da coleta de dados. $\mathrm{O}$ instrumento do controle foi semelhante ao do estudo, incluindo variáveis que não se alteravam em curto espaço de tempo.

Após a finalização da coleta dos dados, foram encontradas 1.084 mulheres de 20 a 60 anos; dessas, 58 (5,3\%) foram classificadas como perdas e recusas, sendo incluídas, no estudo, 1.026 pessoas entrevistadas.

A prevalência de uso de métodos contraceptivos foi investigada entre as mulheres que referiram vida sexual ativa. No momento da entrevista, foi perguntado diretamente se as mulheres mantinham relações sexuais e qual o método anticoncepcional utilizado.

Foram analisadas como variáveis sócio-econômicas: classe econômica segundo a Associação Nacional de Empresas de Pesquisa (ANEP) 8 e escolaridade das mulheres. A classe econômica da ANEP é construída a partir da posse de determinados bens materiais, escolaridade do chefe da família e número de empregados na família. A escolaridade das mulheres foi coletada como anos completos de estudo e categorizada de forma agrupada ( 0 a 4 anos; 5 a 7 anos; 8 a 10 anos; 11 a 14 anos; 15 anos ou mais).

A entrada dos dados foi realizada com dupla entrada, havendo comparação entre elas na 
busca de diminuir erros de digitação, sendo utilizado o programa Epi Info 6.0 (Centers for Disease Control and Prevention, Atlanta, Estados Unidos). A análise foi realizada por meio do programa SPSS versão 11.0 (SPSS Inc., Chicago, Estados Unidos), verificando-se a prevalência dos métodos contraceptivos, de acordo com a idade. Através das razões de prevalências, dos intervalos de confiança e dos testes estatísticos, foram exploradas as associações entre prevalências de uso de anticoncepcional oral e de ligadura tubária com as variáveis sócio-econômicas. Esta análise foi conduzida, inicialmente, em todas as mulheres de 20 a 60 anos com vida sexual ativa e, posteriormente, foi estratificada por idade.

\section{Resultados}

Entre as 1.026 mulheres de 20 a 60 anos entrevistadas com possibilidades de serem incluídas no estudo, $867(84,5 \%)$ relataram manter vida sexual ativa. Dessas 867 mulheres, 240 foram excluídas da análise, pois 153 mulheres não usavam métodos contraceptivos, 32 estavam grávidas no momento da entrevista, 27 estavam na menopausa, 26 tinham sido submetidas à histerectomia, e duas responderam que não podiam ter filhos.

Dessa forma, a análise foi realizada com 627 (61,1\%; IC95\%: 58,1-64,1) mulheres que referiram vida sexual ativa e utilização de algum método contraceptivo, essas foram divididas em dois grupos, de acordo com a idade.

Entre as 840 mulheres de 20 a 49 anos incluídas na amostra, 733 (87,3\%) referiram manter relações sexuais; dessas, 578 (78,8\%; IC95\%: $75,9-81,8)$ usavam algum método contraceptivo e foram incluídas na análise. Nesse primeiro grupo, 282 (48,8\%; IC95\%: 44,7-52,9) mulheres relataram utilizar preferencialmente os anticoncepcionais orais; 108 (18,7\%; IC95\%: 15,521,9) referiram ligadura tubária; 100 (17,3\%; IC95\%: 14,2-20,4), preservativos masculinos; e 42 (7,3\%; IC95\%: 5, 1-9,4) eram usuárias de dispositivos intra-uterinos (Tabela 1 ).

Entre as mulheres de 50 a 60 anos, 186 foram entrevistadas; 134 (72\%) mantinham relações sexuais e foram analisadas; destas, 49 (36,6\%; IC95\%: 28,4-44,7) referiram a utilização de métodos contraceptivos. Nesse grupo, 39 (79,6\%; IC95\%: 68,3-90,9) referiram que a ligadura tubária era o método contraceptivo adotado (Tabela 1).

A análise por idade revelou que a maior prevalência de consumo de anticoncepcionais orais foi na faixa etária de 20 a 24 anos, na qual $77(71,3 \%)$ mulheres relataram usar esse méto-
Tabela 1

Uso de métodos contraceptivos entre as mulheres com vida sexual ativa de 20 a 60 anos. São Leopoldo, Rio Grande do Sul, Brasil, 2003.

\begin{tabular}{|c|c|c|c|c|}
\hline \multirow[t]{2}{*}{ Método contraceptivo } & \multicolumn{2}{|c|}{20 a 49 anos } & \multicolumn{2}{|c|}{50 a 60 anos } \\
\hline & $\mathrm{n}$ & $\%$ & $\mathrm{n}$ & $\%$ \\
\hline Anticoncepcional oral & 282 & 48,8 & - & - \\
\hline DIU & 42 & 7,3 & 2 & 4,1 \\
\hline Coito interrompido & 4 & 0,7 & - & - \\
\hline Preservativo & 100 & 17,3 & 4 & 8,2 \\
\hline Ligadura tubária & 108 & 18,7 & 39 & 79,6 \\
\hline Tabelinha & 2 & 0,3 & - & - \\
\hline $\begin{array}{l}\text { Anticoncepcional oral } \\
\text { e preservativo }\end{array}$ & 1 & 0,2 & - & - \\
\hline Injetável & 20 & 3,5 & - & - \\
\hline Intradérmico & 2 & 0,3 & - & - \\
\hline Vasectomia & 12 & 2,1 & 2 & 4,1 \\
\hline Muco cervical & 1 & 0,2 & - & - \\
\hline Preservativo e injetável & 1 & 0,2 & - & - \\
\hline Anel & 2 & 0,3 & - & - \\
\hline Preservativo e tabela & 1 & 0,2 & - & - \\
\hline Gotas & - & - & 2 & 4,1 \\
\hline Total & 578 & 100,0 & 39 & 100,0 \\
\hline
\end{tabular}

do. Esses valores foram diminuindo progressivamente mostrando associação linear ( $\mathrm{p}<$ $0,0001)$, sendo que, na faixa etária de 45 a 49 anos, 13 (19,7\%) mulheres disseram utilizar anticoncepcional oral; a partir dos 50 anos até os 60 anos, nenhuma mulher relatou o consumo (Tabela 2).

Quanto à prevalência de ligadura tubária, nas mesmas faixas etárias, foram verificados resultados inversos. Esses valores foram aumentando progressivamente também revelando associação linear ( $p<0,0001)$. Se na faixa etária entre 20 e 24 anos nenhuma mulher havia sido esterilizada, entre os 40 a 44 anos, 47 $(40,5 \%)$ mulheres relataram ligadura tubária, observando-se decréscimo na prevalência entre as de 45 e 49 anos (37,9\%). Mas as prevalências continuaram aumentando, alcançando 75,8 e $87,5 \%$ entre as mulheres de 50 a 54 anos e naquelas de 55 a 60 anos, respectivamente (Tabela 2).

A análise da prevalência da anticoncepção oral nas mulheres de todas as idades e as variáveis sócio-econômicas mostraram que as classes de menor inserção econômica tiveram um maior consumo de pílula, inclusive com teste de tendência linear significativo. A variável escolaridade não apresentou diferença estatisticamente significativa entre suas categorias (Tabela 3 ). 
Tabela 2

Uso de anticoncepcionais orais e ligadura tubária por faixas etárias.

São Leopoldo, Rio Grande do Sul, Brasil, 2003.

\begin{tabular}{|c|c|c|c|c|}
\hline \multirow[t]{2}{*}{ Faixa etária (anos) } & \multicolumn{2}{|c|}{$\begin{array}{c}\text { Uso de anti- } \\
\text { concepcional oral* }\end{array}$} & \multicolumn{2}{|c|}{ Ligadura tubária** } \\
\hline & $\mathrm{n}$ & $\%$ & $\mathrm{n}$ & $\%$ \\
\hline $20-24$ & 77 & 71,3 & - & - \\
\hline $25-29$ & 68 & 66,0 & 6 & 5,8 \\
\hline $30-34$ & 51 & 56,7 & 12 & 13,3 \\
\hline $35-39$ & 42 & 44,2 & 18 & 18,9 \\
\hline $40-44$ & 32 & 27,6 & 47 & 40,5 \\
\hline $45-49$ & 13 & 19,7 & 25 & 37,9 \\
\hline $50-54$ & - & - & 25 & 75,8 \\
\hline $55-60$ & - & - & 14 & 87,5 \\
\hline Total & 283 & & 149 & \\
\hline
\end{tabular}

* $p<0,0001$

$\star \star p<0,0001$.
Em relação às variáveis sócio-econômicas e a prevalência de ligadura tubária, foram encontradas associações significativas quanto à classe econômica e escolaridade. A análise nas mulheres de 20 a 60 anos evidenciou que as classes econômicas B, C, D e E apresentavam um menor número de mulheres esterilizadas. Contudo, as mulheres com menor escolaridade apresentavam maior prevalência de ligadura tubária. A associação entre ligadura tubária e escolaridade apresentou tendência linear, ou seja, à medida que diminuía o nível de escolaridade aumentava a prevalência de ligadura tubária, com desvio de linearidade não significativo (Tabela 3).

A associação entre as prevalências de uso de anticoncepcionais orais e de ligadura tubária com classe econômica ou escolaridade poderia estar confundida pela idade. Assim, foi realizada a análise estratificada.

Prevalências de uso de anticoncepcionais orais e de ligadura tubária em relação às variáveis sócio-econômicas. São Leopoldo, Rio Grande do Sul, Brasil, 2003.

\begin{tabular}{|c|c|c|c|c|c|}
\hline Variável & $\mathrm{n}$ & Prevalência (\%) & $\begin{array}{c}\text { Razão de } \\
\text { prevalência }\end{array}$ & $\begin{array}{l}\text { Intervalo de } \\
\text { confiança } 95 \%\end{array}$ & p-valor \\
\hline \multicolumn{6}{|c|}{ Anticoncepcionais orais } \\
\hline \multicolumn{6}{|c|}{ Classe econômica* } \\
\hline Classe A & 48 & $13(27,1)$ & 1,00 & & $<0,01$ \\
\hline Classe B & 162 & $64(39,5)$ & 1,46 & $0,88-2,41$ & \\
\hline Classe C & 262 & $127(48,5)$ & 1,79 & $1,11-2,89$ & \\
\hline Classes D e E & 153 & $78(51,0)$ & 1,88 & $1,15-3,07$ & \\
\hline \multicolumn{6}{|c|}{ Escolaridade (em anos) } \\
\hline 15 ou + & 70 & $28(40,0)$ & 1,00 & & 0,19 \\
\hline $11-14$ & 191 & $89(46,6)$ & 1,16 & $0,84-1,61$ & \\
\hline $8-10$ & 109 & $59(54,1)$ & 1,35 & $0,97-1,89$ & \\
\hline $5-7$ & 150 & $60(40,0)$ & 1,00 & $0,71-1,42$ & \\
\hline $0-4$ & 107 & $47(43,9)$ & 1,10 & $0,77-1,57$ & \\
\hline \multicolumn{6}{|l|}{ Ligadura tubária } \\
\hline \multicolumn{6}{|c|}{ Classe econômica* } \\
\hline Classe A & 48 & $19(39,6)$ & 1,00 & & $<0,03$ \\
\hline Classe B & 162 & $37(22,8)$ & 0,58 & $0,37-0,90$ & \\
\hline Classe C & 262 & $52(19,8)$ & 0,50 & $0,33-0,77$ & \\
\hline Classes D e E & 153 & $39(25,5)$ & 0,64 & $0,41-1,00$ & \\
\hline \multicolumn{6}{|c|}{ Escolaridade (em anos) ${ }^{\star \star}$} \\
\hline 15 ou + & 70 & $10(14,3)$ & 1,00 & & $<0,01$ \\
\hline $11-14$ & 191 & $33(17,3)$ & 1,21 & $0,63-2,32$ & \\
\hline $8-10$ & 109 & $23(21,1)$ & 1,18 & $0,75-2,91$ & \\
\hline $5-7$ & 150 & $45(30,0)$ & 2,10 & $1,13-3,92$ & \\
\hline $0-4$ & 107 & $36(33,6)$ & 2,36 & $1,25-4,43$ & \\
\hline
\end{tabular}

* Teste de tendência linear $<0,01$

$\star *$ Teste de tendência linear $<0,001$. 
Ainda que os intervalos de confiança não tenham revelado diferenças entre as categorias, a análise estratificada mostrou que as prevalências de uso de anticoncepcionais orais entre as mulheres de 20 a 29 anos, nas classes econômicas C, D e E, eram menores do que naquelas de classe A. Porém, na faixa etária de 30 a 39 anos, constatou-se uma inversão na relação, ou seja, as mulheres inseridas nas classes C, D e E apresentaram maior prevalência de uso de contraceptivos orais (Tabela 4).

Esse efeito ficou mais evidente na análise de escolaridade, uma vez que os intervalos de confiança confirmaram as diferenças. As mulheres de 20 a 29 anos com sete ou menos anos de estudo apresentaram prevalências menores do que aquelas com maior escolaridade. Entretanto, na faixa etária entre 30 e 39 anos de idade, as mulheres com menor escolaridade, a partir de dez anos de estudo, apresentaram maiores prevalências de uso de anticoncepcionais orais (Tabela 4). Diante de uma possível modificação de efeito, as variáveis foram transformadas em dicotômicas, sendo aplicado o teste de Mantel-Haenszel, cujo resultado não foi significativo.

Quanto ao consumo de anticoncepcionais orais entre as mulheres de 40 a 49 anos, não foram evidenciadas diferenças quanto às variáveis de classe econômica ou escolaridade ( $\mathrm{Ta}$ bela 4$)$.

Apenas seis mulheres entre 20 e 29 anos de idade haviam sido submetidas à laqueadura tubária, 3 (3,2\%) pertenciam à classe C, e 3 (4,3\%) estavam classificadas nas classes D e E. Quanto à escolaridade, 4 (9,3\%) mulheres apresentavam de 5 a 7 anos de estudo, e 2 (10\%) tinham entre 0 a 4 anos.

A análise estratificada permitiu verificar que, em todas as faixas etárias, a prevalência de ligadura tubária foi maior nas classes $\mathrm{D}$ e E, ao contrário do resultado demonstrado na análise bruta (Tabela 5).

Quanto à variável de escolaridade, em quase todos os estratos etários, exceto entre os $50 \mathrm{e}$ 60 anos, verificou-se que as mulheres com menor nível de estudo apresentaram maior prevalência de ligadura tubária (Tabela 5).

\section{Discussão}

Tradicionalmente, os estudos de saúde reprodutiva incluem as mulheres de 15 a 49 anos. Este estudo estava inserido em projeto com múltiplos desfechos de forma que as mulheres mais jovens foram excluídas. Entretanto, foram incluídas, na análise, mulheres acima de 50 anos, desde que referissem vida sexual ativa. Essa faixa etária, geralmente, está excluída dos estudos sobre consumo de métodos contraceptivos. Do ponto de vista de formulação de atividades em planejamento familiar, sua inclusão se justifica uma vez que os anticoncepcionais orais, por exemplo, estão contra-indicados, em algumas circunstâncias, nas mulheres com mais de 35 anos de idade 9 .

Este estudo transversal foi representativo da população de São Leopoldo, trazendo, como vantagem, a possibilidade de realizar-se inferências e estimativas a partir dos resultados encontrados. Possibilitando, dessa forma, o planejamento e a programação de atividades em saúde a partir da realidade encontrada.

O uso de métodos contraceptivos é influenciado por diversos fatores econômicos, culturais, antropológicos e biológicos. Os estudos que serviram de parâmetros de comparação nessa discussão foram, principalmente, aqueles realizados no Estado do Rio Grande do Sul, tentando diminuir as influências das disparidades regionais.

No presente estudo, verificou-se que quase $80 \%$ das mulheres de até 49 anos de São Leopoldo utilizavam algum método contraceptivo. Esse percentual foi superior ao encontrado em dois estudos transversais de base populacional realizados em Pelotas, Rio Grande do Sul, durante os anos de 1992, 1997 e 1999/2000, nos quais foram encontrados percentuais de $65,7 \%$ 10 e $64,6 \% 7$, respectivamente.

Como era esperado, observou-se, nas mulheres mais jovens, o predomínio de consumo de anticoncepcionais orais, seguido pela ligadura tubária. Os resultados encontrados também foram condizentes com outros estudos realizados no estado. Em Pelotas, os anticoncepcionais orais também foram os principais métodos contraceptivos utilizados pela população feminina. Estudos transversais realizados em 1992 10, 199711 e 20007 mostraram prevalências de uso de anticoncepcionais orais de $66,5 \%, 47,3 \%$ e $55,4 \%$, respectivamente. Em Santa Maria, um estudo transversal envolvendo 355 mulheres de 15 a 44 anos encontrou uma prevalência de $60 \%$ de consumo de anticoncepcionais orais, e $21 \%$ das pessoas entrevistadas estavam esterilizadas 12 .

Os resultados dos estudos de prevalência realizados no Rio Grande do Sul têm apontado para o predomínio da utilização de anticoncepcionais orais e ligadura tubária em detrimento de outros métodos. Contudo, o presente estudo mostrou que, aproximadamente, 1/4 das entrevistadas referiram o uso de DIU ou de 
Prevalência de uso de anticoncepcionais orais conforme classe econômica e escolaridade de acordo com a idade. São Leopoldo, Rio Grande do Sul, Brasil, 2003.

\begin{tabular}{|c|c|c|c|c|c|}
\hline Variável & n & Prevalência (\%) & $\begin{array}{c}\text { Razão de } \\
\text { prevalência }\end{array}$ & $\begin{array}{c}\text { Intervalo de } \\
\text { confiança } 95 \%\end{array}$ & p-valor \\
\hline \multicolumn{6}{|l|}{ 20-29 anos } \\
\hline \multicolumn{6}{|c|}{ Classe econômica } \\
\hline Classe A & 8 & $6(75,0)$ & 1,00 & & 0,49 \\
\hline Classe B & 40 & $31(77,5)$ & 1,03 & $0,67-1,59$ & \\
\hline Classe C & 93 & $63(67,7)$ & 0,90 & $0,59-1,38$ & \\
\hline Classes D e E & 69 & $44(63,8)$ & 0,85 & $0,55-1,32$ & \\
\hline \multicolumn{6}{|c|}{ Escolaridade (em anos)* } \\
\hline 15 ou + & 15 & $13(86,7)$ & 1,00 & & 0,10 \\
\hline $11-14$ & 90 & $63(70,0)$ & 0,81 & $0,64-1,03$ & \\
\hline $8-10$ & 43 & $33(76,7)$ & 0,89 & $0,68-1,15$ & \\
\hline $5-7$ & 43 & $25(58,1)$ & 0,67 & $0,49-0,93$ & \\
\hline $0-4$ & 20 & $11(55,0)$ & 0,63 & $0,41-0,99$ & \\
\hline \multicolumn{6}{|l|}{$30-39$ anos } \\
\hline \multicolumn{6}{|c|}{ Classe econômica* } \\
\hline Classe A & 16 & $5(31,3)$ & 1,00 & & $<0,05$ \\
\hline Classe B & 46 & $17(37,0)$ & 1,18 & $0,52-2,68$ & \\
\hline Classe C & 76 & $45(59,2)$ & 1,89 & $0,89-4,01$ & \\
\hline Classes D e E & 46 & $26(56,5)$ & 1,81 & $0,84-3,91$ & \\
\hline \multicolumn{6}{|c|}{ Escolaridade (em anos)* } \\
\hline 15 ou + & 28 & $9(32,1)$ & 1,00 & & 0,09 \\
\hline $11-14$ & 43 & $18(41,9)$ & 1,30 & $0,68-2,48$ & \\
\hline $8-10$ & 26 & $16(61,5)$ & 1,91 & $1,03-3,55$ & \\
\hline $5-7$ & 38 & $23(60,5)$ & 1,88 & $1,04-3,42$ & \\
\hline $0-4$ & 50 & $27(54,0)$ & 1,68 & $0,93-3,05$ & \\
\hline \multicolumn{6}{|l|}{$40-49$ anos } \\
\hline \multicolumn{6}{|c|}{ Classe econômica } \\
\hline Classe A & 16 & $2(12,5)$ & 1,00 & & 0,7 \\
\hline Classe B & 63 & $16(25,4)$ & 2,03 & $0,52-7,95$ & \\
\hline Classe C & 73 & $19(26,0)$ & 2,08 & $0,54-8,06$ & \\
\hline Classes D e E & 30 & $8(26,7)$ & 2,13 & $0,51-8,88$ & \\
\hline \multicolumn{6}{|c|}{ Escolaridade (em anos) } \\
\hline 15 ou + & 22 & $6(27,3)$ & 1,00 & & 0,25 \\
\hline $11-14$ & 50 & $8(16,0)$ & 0,59 & $0,23-1,49$ & \\
\hline $8-10$ & 31 & $10(32,3)$ & 1,18 & $0,50-2,77$ & \\
\hline $5-7$ & 55 & $12(21,8)$ & 0,80 & $0,34-1,86$ & \\
\hline $0-4$ & 24 & $9(37,5)$ & 1,38 & $0,58-3,24$ & \\
\hline
\end{tabular}

* Teste de tendência linear $<0,05$.

preservativos masculinos, demonstrando, de certa forma, uma razoável diversidade na utilização de métodos. Além disso, a prevalência observada no uso de vasectomia entre a população de São Leopoldo (2,1\%), em termos de comparação, foi três vezes maior do que o percentual de $0,7 \%$ encontrado no estudo realizado em Pelotas em 199210 e mais elevado do que a proporção de $1,6 \%$, verificada entre todas as mulheres usando algum método na Pesquisa Nacional Sobre Demografia e Saúde 4 . Tal achado pode evidenciar o início de uma desejável maior participação dos homens no planejamento familiar.

Vários estudos, no Brasil, têm verificado a prevalência dos métodos contraceptivos, e tem 
Prevalência de ligadura tubária conforme classe econômica e escolaridade de acordo com a idade. São Leopoldo, Rio Grande do Sul, Brasil, 2003.

\begin{tabular}{|c|c|c|c|c|c|}
\hline Variável & $\mathrm{n}$ & Prevalência (\%) & $\begin{array}{c}\text { Razão de } \\
\text { prevalência }\end{array}$ & $\begin{array}{c}\text { Intervalo de } \\
\text { confiança } 95 \%\end{array}$ & p-valor \\
\hline \multicolumn{6}{|l|}{$30-39$ anos } \\
\hline \multicolumn{6}{|l|}{ Classe econômica } \\
\hline Classe A & 16 & $2(12,5)$ & 1,00 & & 0,70 \\
\hline Classe B & 46 & $7(15,2)$ & 1,22 & $0,28-5,27$ & \\
\hline Classe C & 76 & $11(14,5)$ & 1,16 & $0,28-4,73$ & \\
\hline Classes D e E & 46 & $10(21,7)$ & 1,74 & $0,43-7,11$ & \\
\hline \multicolumn{6}{|c|}{ Escolaridade (em anos)* } \\
\hline $11 \mathrm{ou}+$ & 71 & $7(9,8)$ & 1,00 & & 0,052 \\
\hline $8-10$ & 26 & $5(19,2)$ & 1,95 & $0,68-5,61$ & \\
\hline $5-7$ & 38 & $5(13,2)$ & 1,33 & $0,45-3,92$ & \\
\hline $0-4$ & 50 & $13(26,0)$ & 2,64 & $1,13-6,14$ & \\
\hline \multicolumn{6}{|l|}{$40-49$ anos } \\
\hline \multicolumn{6}{|c|}{ Classe econômica } \\
\hline Classe A & 16 & $9(56,3)$ & 1,00 & & $<0,01$ \\
\hline Classe B & 63 & $20(31,7)$ & 0,56 & $0,32-0,99$ & \\
\hline Classe C & 73 & $24(32,9)$ & 0,58 & $0,58-1,01$ & \\
\hline Classes D e E & 30 & $19(63,3)$ & 1,13 & $0,68-1,88$ & \\
\hline \multicolumn{6}{|c|}{ Escolaridade (em anos) } \\
\hline 15 ou + & 22 & $6(27,3)$ & 1,00 & & 0,51 \\
\hline $11-14$ & 50 & $20(40,0)$ & 1,47 & $0,68-3,14$ & \\
\hline $8-10$ & 31 & $10(32,3)$ & 1,18 & $0,50-2,77$ & \\
\hline $5-7$ & 55 & $25(45,5)$ & 1,67 & $0,79-3,50$ & \\
\hline $0-4$ & 24 & $11(45,8)$ & 1,68 & $0,75-3,77$ & \\
\hline \multicolumn{6}{|l|}{$50-60$ anos } \\
\hline \multicolumn{6}{|l|}{ Classe econômica } \\
\hline Classes A e B & 21 & $18(85,7)$ & 1,00 & & 0,31 \\
\hline Classe C & 20 & $14(70,0)$ & 0,82 & $0,58-1,14$ & \\
\hline Classes D e E & 8 & $7(87,5)$ & 1,02 & $0,75-1,40$ & \\
\hline \multicolumn{6}{|c|}{ Escolaridade (em anos) } \\
\hline 15 ou + & 5 & $4(80,0)$ & 1,00 & & 0,96 \\
\hline $11-14$ & 8 & $6(75,0)$ & 0,94 & $0,52-1,70$ & \\
\hline $8-10$ & 9 & $8(88,9)$ & 1,11 & $0,68-1,82$ & \\
\hline $5-7$ & 14 & $11(78,6)$ & 0,98 & $0,59-1,65$ & \\
\hline $0-4$ & 13 & $10(76,9)$ & 0,96 & $0,57-1,63$ & \\
\hline
\end{tabular}

* Teste de tendência linear $<0,05$.

sido demonstrada uma variação de consumo de acordo com a idade das mulheres 13,14,15. Neste estudo, foi verificada a ocorrência na inversão da prevalência entre esses dois métodos em virtude da faixa etária. Assim, quanto mais jovens as mulheres, foi constatado maior consumo de anticoncepcionais orais. Por outro lado, entre as mulheres mais idosas, aumentava o percentual de esterilização. Outros estudos também demonstraram o mesmo efeito 10,10,14.
Ressalta-se a prevalência elevada entre as mulheres de 50 a 60 anos que referiram utilizar o método da ligadura tubária $(79,6 \%)$. Esse achado pode significar o efeito da falta de diversidade de métodos contraceptivos e expressa o efeito de políticas contraceptivas nessa geração, justificando a inclusão dessa faixa etária na análise.

A análise das prevalências do uso de anticoncepcionais orais e de ligadura tubária em 
relação às variáveis sócio-econômicas foi realizada com o intuito de explorar diferenças no acesso aos métodos contraceptivos. Estudos realizados no Rio Grande do Sul têm revelado diferenças negativas em componentes do PAISM de acordo com os níveis sócio-econômicos 16,17,18, confirmando a ainda atual "Lei dos Cuidados Inversos de Hart” 19, cujo enunciado revela que as pessoas com maior risco recebem cuidados de assistência menos qualificados.

A contribuição do presente estudo foi evidenciar uma provável modificação de efeito no consumo de anticoncepcionais orais de acordo com a idade e escolaridade, a partir das razões de prevalência e dos intervalos de confiança. $\mathrm{O}$ teste de Mantel-Haenszel não foi significativo em virtude da falta de poder estatístico necessário para evidenciar o efeito da interação, que talvez pudesse ser demonstrado com um maior tamanho de amostra 20.

Os efeitos observados nas mulheres mais jovens com menor escolaridade e com inserção de classe econômica mais baixa, relacionados à prevalência de anticoncepcionais orais e a ocor- rência de laqueadura tubária podem indicar dificuldades de acesso desde a adolescência.

Em São Leopoldo, a prevalência de laqueadura tubária foi quase sempre mais elevada nas mulheres de escolaridade mais baixa e com menor inserção de classe econômica, não revelando diferenças demonstradas em outros estudos no Estado. Em Pelotas, o estudo de Olinto \& Galvão 11 mostrou que a média de renda familiar em salários mínimos das mulheres esterilizadas era superior à média das não esterilizadas. Outro estudo mostrou que as mulheres com a inserção de classe econômica mais baixa apresentavam menor percentual de ligadura tubária 21.

Assim, os achados da análise em São Leopoldo apontam para uma diversidade na prevalência do uso de métodos contraceptivos, inclusive não fazendo distinção na ocorrência de laqueadura tubária de acordo com variáveis sócio-econômicas, mas ainda revelam a necessidade de propiciar-se escolhas tecnicamente mais adequadas às mulheres mais jovens e com baixa escolaridade.

\section{Resumo}

Foi realizado um estudo transversal de base populacional em São Leopoldo, Rio Grande do Sul, Brasil, com objetivo de analisar o uso de métodos contraceptivos. A amostra envolveu 867 mulheres de 20 a 60 anos com vida sexual ativa. Entre as mulheres que referiram atividade sexual, $627(61,1 \%)$ referiram utilizar algum método contraceptivo. Entre as mulheres de 20 a 49 anos, 48,8\% referiram utilizar anticoncepcionais orais; $18,7 \%$, ligadura tubária; $17,3 \%$, preservativos masculinos; $e$ 7,3\%, dispositivo intra-uterino. Entre as 186 mulheres de 50 a 60 anos que referiram vida sexual ativa, o método mais prevalente foi a ligadura tubária com 79,6\%. Foi observada uma modificação de efeito em relação à prevalência de anticoncepcionais orais, idade e escolaridade, evidenciando uma menor prevalência nas mulheres de 20 a 29 anos e com baixa escolaridade. Assim, os achados da análise em São Leopoldo apontam para uma diversidade na prevalência do uso de métodos contraceptivos, inclusive não fazendo distinção na ocorrência de laqueadura tubária de acordo com variáveis sócio-econômicas, mas ainda revelam a necessidade de propiciar-se escolhas tecnicamente mais adequadas às mulheres mais jovens e com baixa escolaridade.

Anticoncepção; Agentes para Controle da Reprodução; Anticoncepcionais Orais; Esterilização Tubária

\section{Colaboradores}

I. Carreno, J. S. Dias-da-Costa e M. T. A. Olinto participaram da coleta de dados, revisão bibliográfica, análise e discussão do artigo. S. Meneghel colaborou na coleta de dados, análise e discussão do artigo.

\section{Agradecimentos}

Os autores agradecem à Fundação de Amparo à Pesquisa do Estado do Rio Grande do Sul - FAPERGS (via Proad2, n. 02/0645,9), ao Conselho Nacional de Desenvolvimento Científico e Tecnológico - CNPq (Edital Universal 6, n. 473478/200-0) e à Secretaria Municipal de São Leopoldo pelo financiamento e apoio ao projeto de pesquisa. 


\section{Referências}

1. Osis MJMD. Paism: um marco na abordagem da saúde reprodutiva no Brasil. Cad Saúde Pública 1998; 14 Suppl 1:25-32.

2. Ziegel EE, Cranley MS. Enfermagem obstétrica. 8a Ed. Rio de Janeiro: Editora Interamericana; 1985.

3. Hatcher RA, Rinehart W, Blackburn R, Geller JS, Shelton JD. Pontos essenciais da tecnologia de anticoncepção: um manual para pessoal clínico. Baltimore: Population Information Program; 2001.

4. Sociedade Civil Bem-estar Familiar no Brasil. Brasil - Pesquisa Nacional sobre Demografia e Saúde, 1996. Rio de Janeiro: Sociedade Civil Bem-estar Familiar no Brasil; 1997.

5. Teixeira CF. Epidemiologia e planejamento de saúde. Ciênc Saúde Coletiva 1999; 4:287-303.

6. Ross J, Hardee K, Mumford E, Eid S. Contraceptive method choice in developing countries. Int Fam Plan Perspect 2002; 28:32-40.

7. Dias-da-Costa JS, Gigante DP, Menezes AMB, Olinto MTA, Macedo S, Britto MAP, et al. Uso de métodos anticoncepcionais e adequação de contraceptivos hormonais orais na Cidade de Pelotas, Rio Grande do Sul, Brasil: 1992 e 1999. Cad Saúde Pública 2002; 18:93-9.

8. Associação Nacional de Empresas de Pesquisa. Critério de classificação econômica Brasil. http:// www.anep.org.br (acessado em 13/Fev/2004).

9. Oppermann K, Oppermann MLR. Anticoncepção. In: Duncan BB, Schmidt MI, Giugliani ERJ, organizadores. Medicina ambulatorial: condutas de atenção primária baseadas em evidências. v. 1. 3a Ed. Porto Alegre: Artmed Editora; 2004. p. 343-55.

10. Dias-da-Costa JS, D'Elia PB, Moreira MR. Prevalência do uso de métodos contraceptivos e adequação do uso de anticoncepcionais orais na Cidade de Pelotas, Rio Grande do Sul, Brasil. Cad Saúde Pública 1996; 12:339-44.

11. Olinto MT, Galvão LW. Características reprodutivas de mulheres de 15 a 49 anos: estudos comparativos e planejamento de ações. Rev Saúde Pública 1999; 33:64-72.
12. Vilarino JF, Benvegnú LA, Garcia CR, Kraemer CK, Silva DO, Pizutti LT. Estudo epidemiológico da contracepção em Santa Maria. J Bras Ginecol 1998; 108:25-31.

13. Vieira EM. A esterilização de mulheres de baixa renda em região metropolitana do Sudeste do Brasil e fatores ligados a sua prevalência. Rev Saúde Pública 1994; 28:440-8.

14. Schor N, Ferreira AF, Machado VL, França AP, Pirotta KCM, Alvarenga AT, et al. Mulher e anticoncepção: conhecimento e uso de métodos anticoncepcionais. Cad Saúde Pública 2000; 16:37784.

15. Espejo X, Tsunechiro MA, Osis AJD, Duarte GA, Bahamondese L, Souza MH. Adequação do conhecimento sobre métodos anticoncepcionais entre mulheres de Campinas, São Paulo. Rev Saúde Pública 2003; 37:583-90.

16. Dias-da-Costa JS, Victora CG, Barros FC, Halpern R, Horta BL, Manzolli P. Assistência médica materno-infantil em duas coortes de base populacional no Sul do Brasil: tendências e diferenciais. Cad Saúde Pública 1996; 12 Supp1 1:59-66.

17. Quadros CAT, Victora CG, Dias-da-Costa JS. Coverage and focus of a cervical cancer prevention program in southern Brazil. Rev Panam Salud Pública 2004; 16:223-32.

18. Sclowitz ML, Menezes AMB, Gigante DP, Tessaro S. Condutas na prevenção secundária do câncer de mama e fatores associados. Rev Saúde Pública 2005; 39:340-9.

19. Hart JT. Commentary: three decades of the inverse care law. BMJ 2000; 320:18-9.

20. Greenland S, Rothman KJ. Introduction to stratified analysis. In: Rothman KJ, Greenland S, editors. Modern epidemiology. Philadelphia: Lippincott Williams \& Wilkins; 1998. p. 253-79.

21. Dias-da-Costa JS, Olinto MTA. Ligadura tubária: uma representação da iniqüidade do acesso ao sistema de saúde em Pelotas, RS. Rev Ginecol Obstet $1999 ; 10: 130-4$.

Recebido em 02/Mai/2005

Versão final reapresentada em 02/Set/2005

Aprovado em 14/Set/2005 